\title{
A ROBUST ADAPTIVE FUZZY CONTROL APPLIIED TO DISTURBED UNCERTAIN MIMO SYSTEMS
}

\author{
N. ESSOUNBOULI* , A. HAMZAOUI* , K. BENMAHAMMED**, J. ZAYTOON* \\ (*) Laboratoire d'Automatique et de Micro-électronique, UFR Sciences Exactes et Naturelles \\ MOULIN DE LA HOUSSE, B.P. 1039, REIMS CEDEX 2, FRANCE \\ (**) Laboratoire des Systèmes Intélligents, Université Ferhat Abbas Setif, ALGERIE \\ a.hamzaoui@iut-troyes.univ-reims.fr
}

\begin{abstract}
In this paper a robust adaptive fuzzy control for a class of disturbed uncertain multi-input multi-output systems is presented. Adaptive fuzzy systems are used to approximate the system. The effects of both the external disturbances and the approrimation errors are attenuated to a prescribed level using an Ho controller. The adaptation and the control laws are improved to guarantee the boundedness of the variables involved. The proposed approach combines the intelligence of the fuzzy logic and the efficiency of the Hoo technique. $A$ simulation example is presented to lilustrate the tracking performances of this method
\end{abstract}

Keywords-Adaptive fuzzy control, Ho Criterion, Riccati equation.

\section{INTRODUCTION}

The fuzzy control can be an alternative for the complex industrial systems where it is difficult to have a mathematical model. In fact, the linguistic information given by the human expert can be systematically introduced in the controller. Since 1977 , a steam motor controller using fuzzy logic was proposed in [1], and in 1985 a fuzzy controller was used for both a water purification system, and an iron chemical treatment [2]. However, the heuristic design comes without considering the system stability, robustness and performance. To realize a systematic design process, neural and neural-fuzzy networks were employed to construct the fuzzy controllers. By using some training algorithms [3][4], parameters of the fuzzy controller can be obtained automatically. Although this design process is systematic, the global closed-loop system stability may not be guaranteed and the training process is quite time consuming. A fuzzy PID controller was also proposed to control a plant based on the model-free approach. This fuzzy PID controller takes the output error, the derivative of the output error and the integral of the output error as the inputs. Prior knowledge of the plant is not required. Some methodologies on tuning adaptive fuzzy PI, PD and PID controllers can be found in [5][6] and [7]. Based on the freemodel approach, most of the studies were on obtaining the fuzzy controllers, while the stability and robustness of the closed-loop system are seldom considered.

In the case of an uncertain system, the above approaches can not be used. To resolve this problem, adaptive fuzzy approaches are proposed in [8][9]. The linguistic information can be directly incorporated, and both the global stability of the system and the tracking error convergence are guaranteed by a theoretical study. However, the effect of the approximation errors and the external disturbances on the tracking error can not be eliminated efficiently. The subject has attracted the attention of many control researchers. In [10], The authors proposed an adaptive fuzzy algorithm, to approximate the plant, with an $\mathrm{H} \infty$ supervisor to attenuate the effect of both the approximation errors and the external disturbances to a prescribed level. Nevertheless, only a good choice of the initial parameters of the adaptive fuzzy system can guarantee the convergence of the algorithm. A similar approach was proposed in [11], where the adaptation law is improved using a gradient projection technique to maintain the adjustable parameters in a certain region. However, in order to guarantee the existence of the quadratic error integral, the choice of the attenuation level is restricted because of its interaction with the control signal [12]. Furthermore there are no constraints imposed to keep the input function different from zero. Moreover only single input single output (SISO) are studied in these two last references. In [13], a tracking robust adaptive control for a multi-input multi-output (MIMO) system based on a combination of the $\mathrm{H} \infty \mathrm{o}$ tracking theory, VSS control algorithm and fuzzy control design, is proposed. However, as mentioned by the author a partial knowledge of the process is necessary to obtain the control law.

To overcome these problems, an adaptive control algorithm equipped with a projection law and two supervisors is proposed in this work. The first one, $u_{s}$, maintains the system in the controllability region. Hence, the boundedness of all the variables involved, and the existence of the quadratic error integral, are guaranteed. The second one $u_{h}$, attenuates the effect of both the external disturbances and the approximation errors to a prescribed level.

In addition one supposes that no information is available even linguistic, on the system dynamic behavior. The approach advantages with regard to those mentioned previously are:

(i) The possibility to control a highly coupled disturbed uncertain nonlinear MIMO system for which the diagonal elements of the control matrix are not dominants (ii) The variations of the obtained control using this method are less important and has the effect to attenuate the solicitations on the actuators (iii) the resulting control structure can be implemented in real-time due to its simplicity and because the major part of the calculation is performed off-line, (iv) finally, stability, robustness, and convergence of the algorithm are guaranteed by a rigorous mathematical proof.

In section II, the tracking control problem of a disturbed uncertain nonlinear system is formulated. The robust adaptive fuzzy control design is presented in section III. Performances analysis of the proposed approach as well as the introduced improvements are given in section IV. To illustrate the proposed approach, a simulation example of a two-link robot is presented in section $V$. 


\section{Problem Statement}

The $\mathrm{n}^{\text {th }}$ order nonlinear uncertain systems given by the following equations [13]:

$$
\left\{\begin{array}{l}
X^{(n)}=F(x)+G(x) u+d, y=X \\
x=\left[\begin{array}{llll}
X^{T} & \dot{X}^{T} & \cdots & X^{(n-1)^{T}}
\end{array}\right]^{T}
\end{array}\right.
$$

where $F$ and $\mathbf{G}$ are nonlinear continuous functions assumed to be bounded, $u \in \Re^{p}$ and $y \in \Re^{P}$ are the input and output vectors respectively, $\boldsymbol{x}$ is the state vector assumed to be available to measurement, $d$ denotes the external unknown but bounded disturbances (load, measurement noise....).

The control objective is to have the system output $y$ follows a given reference trajectory $y_{r}$. Thus, the tracking error $e=y_{r}-y$ must be as small as possible, and the closed-loop system must be globally stable and robust, i.e., all its parameters are uniformly bounded and the effect of the external disturbances is attenuated to a prescribed level.

If the system is well-known and disturbed, a feedback control law can be applied to it :

$u=G^{-1}(x)\left(-F(x)+y_{\mathrm{I}}^{(\mathrm{n})}+\mathbf{K}^{\mathrm{T}} E-u_{h}\right)$

where the signal $u_{h}$ is used to attenuate the effect of the external disturbances, $\boldsymbol{E}$ and $\mathbf{K}$ denote the error vector and the feedback gain matrix respectively

$$
E=\left[e^{\mathrm{T}} \dot{e}^{\mathrm{T}} \ldots\left(e^{(\mathrm{n}-1)}\right)^{\mathrm{T}}\right]^{\mathrm{T}}, \mathrm{K}=\left[\mathrm{K}_{\mathrm{u}}, \ldots, \mathrm{K}_{1}\right]^{\mathrm{T}}
$$

The dynamic error equation is then:

$e^{(\mathbf{n})}+\mathbf{K}_{1} e^{(\mathrm{n}-1)}+\ldots . .+\mathbf{K}_{\mathrm{n}} \boldsymbol{e}=\boldsymbol{u}_{\mathrm{b}}-\boldsymbol{d}$

In the case of the uncertain plants, and in order to keep the same control structure, two approaches can be considered, one of them consists in approximating the system model, and the second eliminates the model dynamic uncertainties with regard to a reference model. In this paper, the first one is used.

\section{THE ROBUST ADAPTIVE FUZZY CONTROL DESIGN}

\section{A. Fuzzy logic systems (FLS)}

If $\boldsymbol{F}(x)$ and $\mathbf{G}(\boldsymbol{x})$ are unknown and the plant is disturbed, the control law is [14]:

$$
\boldsymbol{u}=\hat{\mathrm{G}}^{-1}(\boldsymbol{x})\left[-\hat{\mathrm{F}}(\boldsymbol{x})+\boldsymbol{y}_{r}^{(n)}+\mathbf{K}^{T} \boldsymbol{E}-\boldsymbol{u}_{\mathrm{h}}\right]
$$

where $\hat{F}(x)$ and $\hat{G}(x)$ are the approximations of $F$ and $\mathbf{G}$ respectively obtained from fuzzy systems.

Using the universal approximator proposed in [11], the function $\hat{F}(x)$ can be written by:

$$
\hat{\boldsymbol{F}}=\xi^{T} \boldsymbol{\theta}_{F}=\left[\begin{array}{llll}
\delta_{1}^{T} \theta_{F-1} & \delta_{2}^{T} \theta_{F-2} & \cdots & \delta_{p}^{T} \theta_{F-p}
\end{array}\right]^{T}
$$

where $\boldsymbol{\theta}_{F}$ is the tunable parameter vector and $\delta_{\mathrm{i}}$ is a $\mathrm{M}^{\text {th }}$ order fuzzy basis function (FBF) and each one of its components can be given as:

$$
\delta_{j}(x)=\left(\prod_{k=1}^{n \times p} \mu_{k}^{j}\right) / \sum_{j=1}^{M}\left(\prod_{k=1}^{n \times p} \mu_{k}^{j}\right) ; \text { where } \mu_{k}^{j} \text { denotes the }
$$

membership function of the $k^{\text {th }}$ fuzzy system input and $M$ is the used fuzzy rules number.

For the function $\hat{\mathrm{G}}(x)$, one has:

$\hat{\mathbf{G}}=\xi^{\mathrm{T}} \Theta_{\mathrm{G}}$

with $\hat{\mathbf{G}}_{i j}=\boldsymbol{\delta}_{\boldsymbol{i}}^{T} \boldsymbol{\Theta}_{G \sim i j}$ for $\mathrm{i}, \mathrm{j}=1, \ldots, \mathrm{p}$
In general, when the number $M$ of the F.B.F. is larger [15], the F.L.S., $\hat{F}\left(x / \theta_{\mathrm{F}}\right)$ et $\hat{\mathrm{G}}\left(\boldsymbol{x} / \boldsymbol{\theta}_{\mathrm{G}}\right)$ can approximate closely any uncertain nonlinear functions $F(x)$ and $G(x)$ by choosing adequately the fuzzy parameters $\boldsymbol{\theta}_{\mathrm{F}}$ and $\boldsymbol{\theta}_{\mathrm{G}}$. In practice, for implementation and computation reasons, the number $M$ of the F.B.F. is chosen as small as possible.

\section{B. Control design}

In order to reduce the number of the F.B.F. and hence the time calculation is decreased, next the fuzzy system tuning laws determination steps will be given, then the control $u_{h}$ which attenuates the approximation error effects as well as the external disturbances.

Rearranging equation (4), one gets:

$y_{\mathrm{r}}^{(\mathrm{n})}=-\mathbf{K}^{\mathrm{T}} E+\hat{F}\left(x / \Theta_{\mathrm{F}}\right)+\hat{G}\left(x / \Theta_{\mathrm{G}}\right) u+u_{\mathrm{h}}$

Combining (1) and (7), and using (5) and (6) the error dynamic equation becomes:

$e^{(n)}=\left[\xi(x)^{\mathrm{T}} \theta_{\mathrm{F}}-F(x)\right]+\left[\xi(x)^{\mathrm{T}} \Theta_{\mathrm{G}}-\mathrm{G}(x)\right] u-K^{\mathrm{T}} E+\left(u_{\mathrm{h}}-d\right)$

which can be written as:

$\dot{\boldsymbol{E}}=\mathbf{A} \boldsymbol{E}+\mathbf{B}\left\{(\hat{\boldsymbol{F}}-\boldsymbol{F})+(\hat{\mathbf{G}}-\mathbf{G}) \boldsymbol{u}+\left(\boldsymbol{u}_{h}-\boldsymbol{d}\right)\right\}$

with :

$\mathbf{A}=\left[\begin{array}{ccccccc}0 & \mathbf{1} & 0 & \cdots & \cdots & 0 & 0 \\ 0 & 0 & \mathbf{I} & \cdots & \cdots & 0 & 0 \\ \vdots & \vdots & \vdots & \vdots & \vdots & \vdots & \vdots \\ 0 & 0 & 0 & 0 & \cdots & 0 & \mathbf{I} \\ -\mathbf{K}_{1} & -\mathbf{K}_{2} & \cdots & \cdots & \cdots & \cdots & -\mathbf{K}_{\mathrm{B}}\end{array}\right], \mathbf{B}=\left[\begin{array}{c}0 \\ 0 \\ \vdots \\ 0 \\ \mathbf{I}\end{array}\right]$

Let $\tilde{\boldsymbol{\theta}}_{\mathrm{F}}$ and $\tilde{\boldsymbol{\theta}}_{\mathrm{G}}$, the estimation errors be defined by: $\tilde{\boldsymbol{\theta}}_{\mathrm{F}}=\boldsymbol{\theta}_{\mathrm{F}}-\boldsymbol{\theta}_{\mathrm{F}}^{*}$ and $\tilde{\boldsymbol{\Theta}}_{\mathrm{G}}=\boldsymbol{\Theta}_{\mathrm{G}}-\boldsymbol{\Theta}_{\mathrm{G}}^{*}$, where $\boldsymbol{\theta}_{\mathrm{F}}^{*}$ and $\boldsymbol{\Theta}_{\mathrm{G}}^{*}$, are optimal estimates of $\boldsymbol{\theta}_{\mathrm{F}}$ and $\boldsymbol{\Theta}_{\mathrm{G}}$.

Let's define the sum of the external disturbances and the approximation errors $\boldsymbol{W}$ as:

$W=\left(F\left(x / \theta_{F}^{*}\right)-F(x)\right)+\left(G\left(x / \theta_{G}^{*}\right)-G(x)\right) u-d$

Equation (8) becomes:

$\dot{E}=\mathrm{A} E+\mathrm{B} u_{\mathrm{h}}+\mathrm{B}\left[\xi^{\mathrm{T}}(x) \tilde{\boldsymbol{\theta}}_{\mathrm{F}}+\xi^{\mathrm{T}}(x) \tilde{\boldsymbol{\theta}}_{\mathrm{G}} u\right]+\mathrm{B} \boldsymbol{W}$

$A$ being a stable matrix, there exists a unique symmetric positive definite matrix $\mathbf{P}$ satisfying the following Riccati equation:

$\mathbf{P A}+\mathbf{A}^{T} \mathbf{P}+\mathbf{Q}-2 \mathbf{P B R}^{-1} \mathbf{B}^{T} \mathbf{P}+\left(1 / \rho^{2}\right) \mathbf{P B B}{ }^{T} \mathbf{P}=0$

where $\mathbf{Q}, \mathbf{R}$ are given symmetric positive definite weighting matrix, and $\rho>0$ is the attenuation level.

The system is robust if it satisfies the following $\mathrm{H}_{\infty}$ criterion:

$$
\begin{aligned}
\int_{0}^{\pi} E^{T} Q E d t & \leq E^{T}(0) \operatorname{P~} E(0)+\frac{1}{\gamma_{F}}\left(\tilde{\Theta}_{F}^{T}(0) \tilde{\Theta}_{F}(0)\right) \\
& +\frac{1}{\gamma_{G}} \operatorname{Trace}\left(\tilde{\Theta}_{G}^{\mathrm{T}}(0) \tilde{\Theta}_{G}(0)\right)+\rho^{2} \int_{0}^{t r} W W^{\mathrm{T}} d t
\end{aligned}
$$

where $\gamma_{F}$ and $\gamma_{G}$ are the learning parameters, and $t r$ is the plant response time.

Choose the following:

$\mathbf{V}=\frac{1}{2} \boldsymbol{E}^{T} \mathbf{P} \boldsymbol{E}+\frac{1}{2 \gamma_{\mathrm{F}}}\left(\tilde{\boldsymbol{\Theta}}_{F}^{T} \tilde{\boldsymbol{\Theta}}_{\mathrm{F}}\right)+\frac{1}{2 \gamma_{G}} \operatorname{Trace}\left(\tilde{\boldsymbol{\Theta}}_{G}^{T} \tilde{\boldsymbol{\Theta}}_{\mathrm{G}}\right)$

(6) as a Lyapunov function. Its time derivative $\dot{V}$ must be negative to ensure the decay of V: 


$$
\begin{aligned}
\dot{\mathbf{V}}= & \frac{1}{2} \dot{\boldsymbol{E}}^{T} \mathbf{P} \boldsymbol{E}+\frac{1}{2} \boldsymbol{E}^{T} \mathbf{P} \dot{\boldsymbol{E}}+\frac{1}{2 \gamma_{F}}\left(\dot{\boldsymbol{\theta}}_{F}^{T} \tilde{\boldsymbol{\theta}}_{F}+\tilde{\boldsymbol{\theta}}_{F}^{T} \dot{\boldsymbol{\theta}}_{F}\right) \\
& +\left(1 / 2 \gamma_{G}\right) \operatorname{Trace}\left(\dot{\boldsymbol{\Theta}}_{G}^{T} \tilde{\boldsymbol{\theta}}_{G}+\tilde{\boldsymbol{\Theta}}_{G}^{T} \dot{\boldsymbol{\theta}}_{G}\right)
\end{aligned}
$$

Since $\boldsymbol{\Theta}_{\mathrm{F}}^{*}$ and $\boldsymbol{\Theta}_{\mathrm{G}}^{*}$ are constants, then: $\dot{\tilde{\boldsymbol{\Theta}}}_{F}=\dot{\boldsymbol{\Theta}}_{F} ; \dot{\tilde{\boldsymbol{\Theta}}}_{G}=\dot{\boldsymbol{\Theta}}_{G}$ Substituting (10) in (14), and using (11) one gets: $2 \dot{\mathbf{V}}=-E^{T} \mathrm{QE}+2 E^{T} \mathrm{~PB}\left(\mathbf{R}^{-1} \mathbf{B}^{T} \mathrm{P} E+u_{h}\right)+2 E^{T} \mathrm{~PB} \xi^{T} \tilde{\boldsymbol{\theta}}_{G}$ $-\frac{1}{\rho^{2}} E^{T} \operatorname{PBB}^{T} \mathrm{P} E+2 E^{T} \mathrm{~PB} W+\frac{2}{\gamma_{G}} \sum_{\mathrm{i}=1}^{\mathrm{p}} \sum_{\mathrm{j}=1}^{\mathrm{p}}\left(\tilde{\theta}_{G_{y}}^{T} \dot{\theta}_{G_{j i}}\right)$

$$
+\tilde{\mathbf{\Theta}}_{F}^{T}\left(2 \xi \mathbf{B}^{T} \mathbf{P} E+\frac{2}{\gamma_{F}} \dot{\boldsymbol{\theta}}_{F}\right)
$$

If the control law $u_{h}$, and the parameters adaptation laws of $\boldsymbol{\theta}_{\mathrm{F}}$ and $\boldsymbol{\Theta}_{\mathrm{G}}$ are chosen as:

$$
\begin{aligned}
& u_{\mathrm{h}}=-\mathbf{R}^{-1} \mathbf{B}^{\mathrm{T}} \mathbf{P} E \\
& \dot{\boldsymbol{\theta}}_{\mathrm{F}}=-\gamma_{\mathrm{F}} \xi \mathbf{B}^{\mathrm{T}} \mathbf{P} E
\end{aligned}
$$

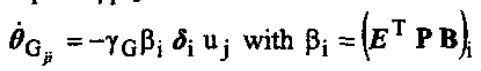

the equation (15) is simplified to the following form:

$$
\begin{aligned}
2 \dot{\mathrm{V}}= & -E^{\mathrm{T}} \mathbf{Q} E-\left(\frac{\mathbf{B}^{\mathrm{T}} \mathbf{P} E}{\rho}-\rho W\right)^{\mathrm{T}}\left(\frac{\mathbf{B}^{\mathrm{T}} \mathbf{P} E}{\rho}-\rho W\right)(17) \\
& +\rho^{2} W^{\mathrm{T}} W
\end{aligned}
$$

Since the term $\left[\left(\left(\mathbf{B}^{T} \mathbf{P} E / \rho\right)-\rho W\right)^{T}\left(\left(\mathbf{B}^{T} \mathrm{P} E / \rho\right)-\rho W\right)\right]$ is positive, then:

$$
\dot{\mathrm{v}} \leq \frac{-E^{\mathrm{T}} \mathrm{Q} E}{2}+\frac{\rho^{2} W^{\mathrm{T}} W}{2}
$$

Integrating the above inequality from $t=0$ to $t$, to get:

$$
\begin{array}{r}
\int_{0}^{\pi} E^{T} \mathbf{Q} E d t \leq E^{T}(0) P E(0)+\frac{1}{\gamma_{F}}\left(\tilde{\Theta}_{F}^{T}(0) \tilde{\Theta}_{F}(0)\right) \\
+\frac{1}{\gamma_{G}} \operatorname{Trace}\left(\tilde{\Theta}_{G}^{\mathrm{T}}(0) \tilde{\boldsymbol{\Theta}}_{G}(0)\right)+\rho^{2} \int_{0}^{\pi} W W^{\mathrm{T}} d t
\end{array}
$$

which represents the $\mathrm{H}_{\infty}$ criterion given in (12).

Remark

The Riccati equation (11) has a unique symmetric definite positive solution $P$, if and only if $2 \rho^{2} I \geq R$. A robust tracking performance with an arbitrarily attenuation level of $W(t)$ can be guaranteed with this approach by a good choice of the weighting matrix $\mathbf{R}$. However, if the attenuation level is very small, the control signal $u_{\mathrm{h}}(\mathrm{t})$ can be larger. Hence, there is a trade-off between $\rho$ and $u_{\mathrm{h}}(\mathrm{t})$.

If the control $u_{\mathrm{h}}(\mathrm{t})$ is also concerned, the same method can be used and the Riccati equation should be modified as:

$$
\mathbf{P A}+\mathbf{A}^{T} \mathbf{P}+\mathbf{Q}-\mathbf{P B R}^{-1} \mathbf{B}^{T} \mathbf{P}+\left(1 / \mathbf{\rho}^{2}\right) \mathbf{P B B}{ }^{T} \mathbf{P}=0
$$

and the $H \infty$ becomes :

$$
\begin{aligned}
\int_{0}^{t r}\left(E^{T} \mathbf{Q} E+u_{h}^{T} \mathbf{R} u_{h}\right) d t & \leq E^{T}(0) \mathbf{P} E(0)+\frac{1}{\gamma_{F}}\left(\tilde{\boldsymbol{\Theta}}_{F}^{\mathbf{T}}(0) \tilde{\boldsymbol{\Theta}}_{F}(0)\right) \\
& +\frac{1}{\gamma_{G}} \operatorname{Trace}\left(\tilde{\boldsymbol{\Theta}}_{G}^{\mathrm{T}}(0) \tilde{\boldsymbol{\Theta}}_{G}(0)\right)+\rho^{2} \int_{0}^{t r} \boldsymbol{W} W^{\mathrm{T}} d t
\end{aligned}
$$

\section{CONVERGENCE ANALYSIS}

A stable robust adaptive fuzzy controller is developed, using an $\mathrm{H} \infty$ technique and the Lyapunov approach. The next task, is to verify the convergence of the tracking error toward zero. In fact, to have $\dot{V}$ negative, $\left(\rho^{2} W^{T} W\right) / 2$ should be small. Since The external disturbances $d$ are assumed to be bounded, it's left to check that the minimal approximation errors are also bounded. Unfortunately the adaptation law (16) can not guarantee the boundedness of the tuned parameters $\boldsymbol{\theta}_{\mathrm{F}}$ and $\boldsymbol{\theta}_{\mathrm{G}}$.

Rearranging equation (18) we get:

$$
\begin{aligned}
& \left(\lambda_{\min }(\mathbf{Q}) / 2\right)|E|^{2} \leq-\dot{\mathbf{V}}+\left(\rho^{2} / 2\right)|W|^{2} \\
\Rightarrow & |E|^{2} \leq\left(2 /\left(\lambda_{\min }(\mathbf{Q}) / 2\right)\right)\left(-\dot{\mathbf{V}}+\left(\rho^{2} / 2\right)|W|^{2}\right)
\end{aligned}
$$

where $\lambda_{\text {min }}(Q)$ denotes the minimal eigenvalue of $\mathbf{Q}$. integrating the above inequality from 0 to $t$, we obtain: $\int_{0}^{t}|E(\tau)|^{2} \mathrm{~d} \tau \leq \mathrm{a}+\mathrm{b} \int_{b}^{p}|W|^{2}(\tau) \mathrm{d} \tau$

with: $a=\frac{2}{\lambda_{\min }(\mathrm{Q})}\left(\mathrm{V}(0)-\sup _{\mathrm{t} \geq 0} \mathrm{~V}(\mathrm{t})\right)$ and $\mathrm{b}=\frac{\rho^{2}}{\lambda_{\min }(\mathrm{Q})}$

The inequality (22) is satisfied if and only $\lambda_{\min }(Q)$ is different from zero; this is verified due to the fact that $Q$ is given by the designer. Using the BARBALAT lemma [9], if "a" is bounded and the integral $\int_{0}^{t}|W|^{2}(\tau) d \tau$ exists for $\mathrm{t} \rightarrow \infty$, then $\lim _{\mathrm{t} \rightarrow \infty} E(\mathrm{t})=0$.

The external disturbances $\boldsymbol{d}$ and the functions $\boldsymbol{F}$ and $\mathbf{G}$ are assumed to be bounded, hence the approximation functions $\hat{\boldsymbol{F}}$ and $\hat{\mathbf{G}}$ must be bounded. Therefore, the adaptation law given by (16) must be modified.

\section{A. The modified adaptation law}

The adaptation law can be modified using a gradient projection algorithm [16] to maintain the adjustable parameters in certain constraint region defined by:

$$
\begin{aligned}
& \Omega_{F}=\left\{\theta_{F}:\left|\theta_{F}\right| \leq M_{F}<\infty\right\} \\
& \Omega_{\mathbf{G}_{\mathbf{i j}}}=\left\{\boldsymbol{\theta}_{\mathbf{G}_{\mathbf{i j}}}:\left|\boldsymbol{\theta}_{\mathbf{G}_{\mathbf{i j}}}\right| \leq M_{\mathbf{G}_{\mathbf{i j}}}<\infty,\left(\boldsymbol{\theta}_{\mathbf{G}_{\mathbf{i j}}}\right)_{k} \geq \varepsilon_{\mathbf{G}_{\mathbf{i j}}}\right\}, \mathrm{i}, \mathrm{j}=1, \ldots, \mathrm{p} ;
\end{aligned}
$$

where $M_{F}, M_{\mathrm{GIJ}}$ and $\varepsilon_{\mathrm{Gl}}$ are constants given by the designer. Note that $\varepsilon_{G \text { ij }}$ is used to guarantee the inversion of the matrix $\hat{\mathbf{G}}$.

The modified adaptation law is given by:

$\dot{\theta}_{F}=\left\{\begin{aligned} &-\gamma_{F} \zeta(x) B^{\mathrm{T}} \mathrm{P} E \text { if }\left(\left|\theta_{F}\right|<\mathrm{M}_{\mathrm{F}}\right) \\ & \text { or }\left(\left|\theta_{F}\right|=\mathrm{M}_{\mathrm{F}} \text { and } \theta_{F}^{\mathrm{T}} \zeta(x) \mathrm{B}^{\mathrm{T}} \mathrm{P} E \geq 0\right) \\ & \gamma_{F} \operatorname{Pr}\left\{\zeta(x) \mathrm{B}^{\mathrm{T}} \mathrm{P} E\right\}_{\text {if }}\left(\left|\theta_{F}\right|=\mathrm{M}_{\mathrm{F}} \text { and } \theta_{F}^{\mathrm{T}} \zeta(x) \mathrm{BB}^{\mathrm{T}} \mathrm{P} E<0\right)\end{aligned}\right.$

where $\operatorname{Pr}\left\{-\zeta(x) \mathrm{B}^{T} \mathbf{P} E\right\}=-\zeta(x) \mathbf{B}^{T} \mathbf{P} E+\zeta(x) \frac{\theta \theta^{T}}{|\theta|^{2}} \mathbf{B}^{T} \mathbf{P} E$ 


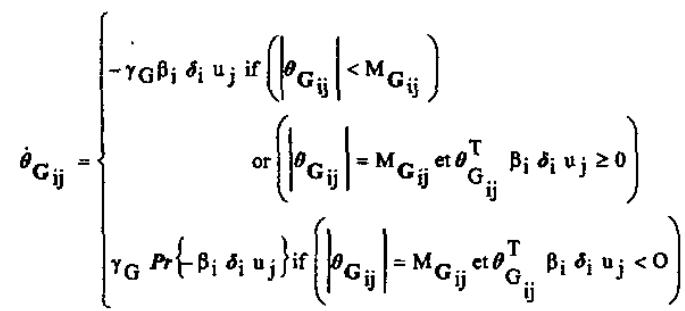

if an element $\left(\theta_{\mathbf{G i j}}\right)_{k}=\varepsilon_{\mathrm{Gij}}$, then

$\left(\dot{\theta}_{\mathrm{Gij}}\right)_{k}=\left\{\begin{array}{cc}\left(\dot{\theta}_{\mathrm{Gij}}\right)_{k} & \text { if } \theta_{\mathrm{G}_{\mathrm{ij}}^{\mathrm{T}}}^{\mathrm{B}} \beta_{\mathrm{i}} \delta_{\mathrm{i}} u_{\mathrm{j}}<0 \\ 0 & \text { if } \theta_{\mathrm{G}_{\mathrm{ij}}^{\mathrm{T}}}^{\mathrm{T}} \beta_{\mathrm{i}} \delta_{\mathrm{i}} \mathrm{u}_{\mathrm{j}} \geq 0\end{array}\right.$

$B$. The control supervisor $u_{s}$

In this section, the control law should be modified to guarantee the boundedness of the term " $a$ " in the equation (22). To do that, one has to bound the Lyapunov function.

Consider the following Lyapunov function

$\mathrm{V}=\frac{1}{2} E^{\mathrm{T}} \mathrm{P} E$ supposed to have the upper bound $\overline{\mathrm{V}}<\infty$.

So one can write :

$$
\begin{gathered}
V \geq \frac{1}{2} \lambda_{\min }(\mathbf{P})|E|^{2} \Rightarrow\left(2 \mathrm{~V} / \lambda_{\min }(\mathbf{P})\right)^{1 / 2} \geq|E| \geq|x|-\left|y_{r}\right| \\
\Rightarrow|x| \leq\left|y_{r}\right|+\left(2 \mathrm{~V} / \lambda_{\min }(\mathbf{P})\right)^{1 / 2}
\end{gathered}
$$

Then, If one wishes that $|x| \leq M_{x}$, one gets :

$$
\bar{V}=\frac{\lambda_{\min }(\mathrm{P})}{2}\left(\mathrm{M}_{x}-\sup _{\mathrm{t} \geq 0}\left(\left|y_{\mathrm{r}}\right|\right)\right)^{2}
$$

Once $\bar{V}$ is defined, the control signal $\boldsymbol{u}_{s}$ will be determined. The last Lyapunov function is retaken and derived over the dynamic error trajectory :

$$
\dot{V}=\frac{1}{2} \dot{E}^{\mathrm{T}} \mathrm{P} E+\frac{1}{2} E^{\mathrm{T}} \mathrm{P} \dot{E}
$$

Using equation (8), one obtain :

$$
\begin{aligned}
2 \dot{\mathrm{V}}= & \left(\mathrm{A} E+\mathrm{B}\left\{[\hat{F}-F]+[\hat{G}-\mathrm{G}] u+\left(u_{\mathrm{h}}-d\right)\right\}\right)^{\mathrm{T}} \mathrm{P} E \\
& E^{\mathrm{T}} \mathrm{P}\left(\mathrm{A} E+\mathrm{B}\left\{[\hat{F}-F]+[\hat{G}-\mathrm{G}] u+\left(u_{\mathrm{h}}-d\right)\right\}\right) \\
2 \dot{\mathrm{V}}= & E^{\mathrm{T}}\left(\mathrm{A}^{\mathrm{T}} \mathrm{P}+\mathrm{PA}\right) E \\
& +2 E^{\mathrm{T}} \mathrm{PB}\left(\left\{[\hat{F}-F]+[\hat{G}-G] u+\left(u_{\mathrm{h}}-d\right)\right\}\right)
\end{aligned}
$$

the Riccati equation (11) gives :

$$
\begin{aligned}
2 \dot{\mathrm{V}}=- & E^{\mathrm{T}} \mathrm{Q} E+2 E^{\mathrm{T}} \mathrm{PB}\left(\mathrm{R}^{-1} \mathbf{B}^{\mathrm{T}} \mathrm{P} E+u_{\mathrm{h}}\right) \\
& -\left(\frac{E^{\mathrm{T}} \mathrm{PB}}{\rho}-\rho d\right)^{\mathrm{T}}\left(\frac{E^{\mathrm{T}} \mathrm{PB}}{\rho}-\rho d\right) \\
& \left.+\rho^{2} d^{T} d+2 E^{\mathrm{T}} \mathrm{PB}(\hat{F}-F]+[\hat{\mathrm{G}}-\mathrm{G}] u\right)
\end{aligned}
$$

which can be simplified in the following way:

$$
\left.\dot{V} \leq-\frac{1}{2} E^{T} Q E+\frac{\rho^{2}}{2} d^{T} d+E^{\mathrm{T}} \mathrm{PB}(\hat{F}-F]+[\hat{G}-\mathrm{G}] u\right)
$$

But the sign of the expression $\left.E^{\mathrm{T}} \mathbf{P B}(\hat{F}-F]+[\hat{G}-\mathbf{G}] \mu\right)$ cannot be determined, then the negative sign of $\dot{V}$ cannot be guaranteed. If one has the case where $\dot{V} \geq 0$ and $V \geq \bar{V}$, then an additional control signal must be used to insure that $\dot{\mathrm{V}} \leq 0$ when $V \geq \bar{V}$.

If the global control is taken to be : $\boldsymbol{u}_{f}=u+\boldsymbol{u}_{s}$ then equation (8) can be rewritten in the form :

$\dot{E}=\mathbf{A} E+B\left\{\{\hat{F}-F]+[\hat{G}-\mathbf{G}] u-G u_{\mathrm{s}}+\left(u_{\mathrm{h}}-d\right)\right\}$

resulting in :

$2 \dot{\mathrm{V}}=-E^{\mathrm{T}} \mathrm{Q} E+2 E^{\mathrm{T}} \mathbf{P B}\left(\mathbf{R}^{-1} \mathbf{B}^{\mathrm{T}} \mathrm{P} E+u_{\mathrm{h}}\right)$

$$
\begin{aligned}
& -\left(\frac{E^{\mathrm{T}} \mathrm{PB}}{\rho}-\rho d\right)^{\mathrm{T}}\left(\frac{E^{\mathrm{T}} \mathrm{PB}}{\rho}-\rho d\right) \\
& \left.+\rho^{2} d^{\mathrm{T}} d+2 E^{\mathrm{T}} \mathrm{PB}(\hat{F}-F]+[\hat{\mathrm{G}}-\mathrm{G}] u-\mathrm{G} u_{s}\right)
\end{aligned}
$$

Let

$G_{m}$ minimum value of $G, G^{M}$ maximum value of $G$, and $\mathrm{F}^{\mathrm{M}}$ maximum value of $\mathrm{F}$.

If the control signal $\boldsymbol{u}_{\mathrm{s}}$ is chosen in the form :

$u_{S}=G_{m}^{-1} \operatorname{sign}\left(E^{T} \mathbf{P B}\right)\left[|\hat{F}|+F^{M}+|\hat{G} u|+\left|G^{M} u\right|\right]$

then the following inequality is checked :

$2 \dot{\mathrm{V}} \leq-E^{\mathrm{T}} \mathrm{Q} E+\rho^{2} d^{T} d$

Hence, for a given $\rho$, one will have :

$2 \dot{\mathrm{V}} \leq-\boldsymbol{E}^{\mathrm{T}} \mathrm{Q} E$

It has shown that the control $\left(u_{s}\right)$ allows the tracking error convergence toward zero.

The equation (4) can be written in the form:

$u=u_{1}+u_{2}$ with :

$\boldsymbol{u}_{I}=\hat{\mathbf{G}}^{-1}(\boldsymbol{x})\left[-\hat{\boldsymbol{F}}(x)+y_{r}^{(n)}+\mathbf{K}^{T} E\right]$ et $\boldsymbol{u}_{2}=-\hat{\boldsymbol{G}}^{-1}(x) u_{\mathrm{b}}$

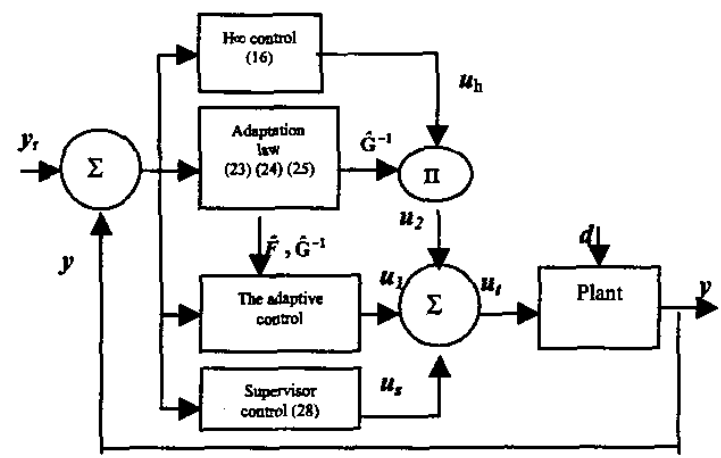

Fig. 1. The control scheme

The figure 1 represents the proposed control scheme. The updating parameters $\boldsymbol{\theta}_{\mathrm{F}}$ and $\boldsymbol{\Theta}_{\mathrm{G}}$ allows the approximations $\hat{\boldsymbol{F}}\left(\boldsymbol{x} / \boldsymbol{\theta}_{\mathrm{F}}\right)$ and $\hat{\mathrm{G}}\left(\boldsymbol{x} / \boldsymbol{\theta}_{\mathrm{G}}\right)$ which will be used to generate the control $u_{1}$. The supervisor control $\left(u_{2}\right)$ attenuates the effects of both the external disturbances and the approximation errors to a prescribed level $\rho$. The second supervisor control $\left(\boldsymbol{u}_{\mathrm{s}}\right)$ allows to bound the plant states and to keep them in the controllability zone.

The control structure such that the one given in figure 1, shows the approach implementation simplicity. In fact, the global control is given by a simple combination of the outputs of the four parallel blocks; contrarily to the approaches cited in to the introduction where the global control is given by a combination of the series blocks. 


\section{SIMULATIONS}

To validate the proposed approach, consider a two-link robot, with masses $m_{1}$ and $m_{2}$, and of lengths $l_{1}$ and $l_{2}$, as shown in figure 2.

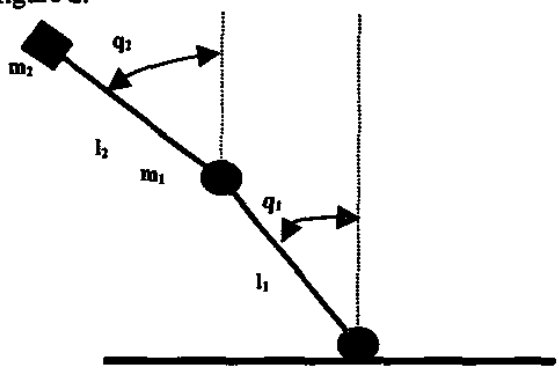

Fig. 2. The two-link robot

Let $q=\left[q_{1} q_{2}\right]^{\mathrm{T}}$ be the rotation angle vector of the articulations with respect to the vertical axe. Using the Lagrange equation [17], the system dynamic equation can be written :

$$
\mathbf{M}(q) \ddot{q}+\mathbf{C}(q, \dot{q}) \dot{q}+\mathbf{G}(q)=\Gamma+d
$$

where $\dot{q}, \ddot{q}$ are the velocity and the acceleration vectors, respectively,

$\mathbf{M}(q)$ is the inertia matrix, which is symmetric, positive definite:

$$
\begin{aligned}
& \mathbf{M}(q)=\left[\begin{array}{cc}
\left(m_{1}+m_{2}\right) y_{1}^{2} & m_{2} l_{1} l_{2} \cos \left(q_{1}-q_{2}\right) \\
m_{2} l_{1} l_{2} \cos \left(q_{1}-q_{2}\right) & m_{2} l_{2}^{2}
\end{array}\right] \\
& \mathbf{C}(q, \dot{q})=m_{2} l_{1} l_{2} \sin \left(q_{1}-q_{2}\right)\left[\begin{array}{cc}
0 & \dot{q}_{2} \\
\dot{q}_{1} & 0
\end{array}\right]: \text { the Coriolis matrix } \\
& \mathbf{G}(q)=\left[\begin{array}{c}
-\left(m_{1}+m_{2}\right) y_{1} g \sin \left(q_{1}\right) \\
-m_{2} l_{2} g \sin \left(q_{2}\right)
\end{array}\right]: \text { the gravitational vector }
\end{aligned}
$$

$\Gamma \in \mathfrak{R}^{2}$ is torque vector, and $d \in \mathfrak{R}^{2}$ the external disturbances.

Equation (29) can be written in the form (1) as:

$$
\ddot{q}=-M^{-1}(q)(C(q, \dot{q}) \dot{q}+\mathbf{G}(q))+M^{-1}(q) \Gamma+M^{-1}(q) d
$$

then the proposed approach can be used.

For the simulation, consider a robot with the following characteristics: $m_{1}=5 \mathrm{Kg}, \mathrm{m}_{2}=2.5 \mathrm{Kg}, \mathrm{l}_{1}=\mathrm{l}_{2}=0.5 \mathrm{~m}$. Suppose that the system is subject to the extemal disturbances $\mathrm{d}_{1}=\mathrm{d}_{2}=0.01 \sin (2 \mathrm{t})$.

The robot is assumed to be at rest, and the articulation initial positions are $q_{1}=1.5 \mathrm{rad}$ and $q_{2}=1.2 \mathrm{rad}$.

The solution of the Riccati equation, using the "Are" function of the Matlab environment, is given by:

$\mathbf{P}=\left[\begin{array}{llll}8.1250 & 0.0000 & 2.7500 & 0.0000 \\ 0.0000 & 8.1250 & 0.0000 & 2.7500 \\ 2.7500 & 0.0000 & 2.6250 & 0.0000 \\ 0.0000 & 2.7500 & 0.0000 & 26250\end{array}\right]$

for $\mathbf{K}_{1}=\operatorname{diag}(1,1), \mathbf{K}_{2}=\operatorname{diag}(2,2), Q=\operatorname{diag}\left(\begin{array}{llll}5.5 & 5.5 & 5 & 5\end{array}\right)$ and $\mathbf{R}=2 . \rho^{2} \mathbf{I}_{(2 \times 2) \text {. }}$.

To approximate the system, consider five gaussian membership functions for the inputs: $\mathrm{q}_{1}, \mathrm{q}_{2}, \dot{\mathrm{q}}_{1}, \dot{\mathrm{q}}_{2} . F$ is a function which depends on the four previous inputs. At the beginning, all possible combinations were used which has generated 625 rules. Since $F$ is more dependent on the angular positions on one hand, and the effect of the approximation can be attenuated on the other hand, only 50 rules can be used.
Then, we obtain the same result and the computation time is considerably reduced.

The robot initial position was chosen far away from the desired trajectory, and the external disturbances were taken sufficiently large to illustrate the efficiency of the proposed approach.

Several simulations were done for different values of $\rho$. The results presented below are obtained with an attenuation of $90 \%, \rho=0.1$. The response and the reference trajectories are depicted in figures 3 and 4 , which show that there is a good tracking performance. Figure 5 gives the applied torques, the soliciting at the input levels are acceptable even in the transient state. The quadratic errors are depicted in figure 6. Figure 7 gives the quadratic error integral, which is constant and hence it allows to conclude that there is a good convergence.

Several comparisons with the classical methods have been done. The obtained results show that the proposed approach allows to get better performances. For example and for lack of space, we give only results gotten for the same example with a control law deducted from a nominal model [18]. The effect of both the external disturbances and the uncertainties is attenuated by the $\mathrm{H}_{\infty}$ signal, but the initial values of the applied torques are more important $\left(u_{1}=-363 \mathrm{~N} . \mathrm{m}, u_{2}=-145 \mathrm{~N} . \mathrm{m}\right.$ for classical method, and $u_{1}=-296 \mathrm{~N} . \mathrm{m}, u_{2}=-66 \mathrm{~N} . \mathrm{m}$ for the proposed method). Furthermore, no mathematical model of the process is required to determine the control law developed in this work. But only a partial knowledge about the dynamic behavior of the system is sufficient.

\section{CONCLUSION}

In this paper, a robust adaptive fuzzy approach to control disturbed uncertain non linear MIMO systems is proposed. Adaptive fuzzy systems are used to approximate the plant. The effects of the approximation enrors and the external disturbances are attenuated to a prescribed level by an $H_{\infty}$ controller. The closed loop system global stability in Lyapunov sense is guaranteed and proved analytically. The adaptive algorithm convergence is insured by a gradient type projection and a supervisor control signal. The approach advantage is its implementation simplicity. In fact, several computation steps can be done off line. Notice that no information about the system dynamic behavior is needed for the adaptive algorithm design. If the system bounds are known, then the convergence adaptation law can be improved considerably. The only constraint is that the weighting matrix $\mathbf{R}$ choice must insure the solvability of the Riccati equation. The smaller $\rho$ is, the more important is the control signal in the transient state; there must

be a trade off between the control signal magnitude and the attenuation level.

\section{REFERENCES}

[1] P.J. King and E. P. Mamdani, "the application of fuzzy control systems to industrial process," Automatica, vol. 13, no. 3, pp. 235-242, 1977.

[2] T. Takagi and M. Sugeno, "Identification of systems and its applications to modeling and control," IEEE Trans. Syst., Man, Cybern. vol. SMC-15, no. 1, pp. 116-132, 1985. 
[3] A.Blanco, M.Delgado and I.Requena, " A leaning procedure to identify weighted rules by neural networks," Fuzzy Sets Syst. no. 69, pp. 29-36, 1995.

[4] C.T. Lin and Y.C. Lu, "A neural fuzzy system with linguistic teaching signals," IEEE Trans. Fuzzy Syst., vol. 3, no. 2, pp. 169-189, 1995.

[5] Z.Y. Zhao, M. Tomizuka, and S. Isaka, "Fuzzy gain scheduling of PID controllers," IEEE Trans. Syst., Man, Cybern., vol. 23, no. 5, pp. 1392-1398, 1993.

[6] W.Z. Qianoa and M. Mizumoto, "PID type fuzzy controller and parameters adaptive method," Fuzzy Sets Syst., vol. 78, pp. 23-35, 1996.

[7] G.K.I. Mann, B.G. Hu, and R.G. Gosine, "Analysis of direct action fuzzy PID controller structurs," IEEE Trans. Syst., Man, Cybern., vol. 29, no. 3, pp. 371-388, 1999.

[8] C.Y. Su and Y. Stepaneko," Adaptive control of a class of nonlinear systems with fuzzy logic," IEEE Trans. Fuzzy Syst., vol. 2, no. 4, pp. 285-294, 1994.

[9] L-X. Wang, " A course in fuzzy systems and control", Prentice Hall PTR ; Upper Saddle, N.J., U.S.A., 1997.

[10] A. Hamzaoui, J. Zaytoon and A. Elkari, "adaptive fuzzy control for uncertain nonlinear systems," In Proc. Of IFAC Workshop on Control Applications of Optimization, pp. 149-152, Saint-Petersburg, Russia.

[11] B-S. Chen, C-H. Lee and Y6C. Chang, " $\mathrm{H}_{\infty}$ tracking design of uncertain nonlinear SISO systems : Adaptive fuzzy approach," IEEE Trans. Fuzzy Syst., vol. 4, no. 1, pp. 32-43, 1996.

[12] H.J. Kang, $\mathbf{H}$. Lee and M. Park, " Comments on $\mathbf{H}_{\infty}$ tracking design of uncertain nonlinear SISO systems : Adaptive fuzzy approach," IEEE Trans. Fuzzy Syst., vol. 6, no. 4, pp. 605-606, 1998.

[13] Y-C. Chang, " Robust tracking control for nonlinear MIMO systems via fuzzy approaches," Automatica, vol. 36 , pp. 1535-1545, 2000.

[14] S. Sastry and N. Bodson" Adaptive Control: Stability, Convergence and Robustness," Englewood Cliff's, N.J. : Prentice Hall, U.S.A., 1989.

[15] L-X. Wang, " Adaptive fuzzy systems and control," Englewood Cliff's, N.J. : Prentice Hall, U.S.A., 1994.

[16] G.C. Goodwin, and D.Q. Mayne, "a parameter estimation perspective of continuous time model reference adaptive control," Automatica, vol. 23, pp. 57-70, 1987.

[17] J.J. Craig, "Adaptive control of mechanical manipulators," A-W. Publishing Company, 1988.

[18] J.J. Slotine and W.Li , "Applied nonlinear control," Englewood Cliffs, NJ: Prentice-Hall, 1991.

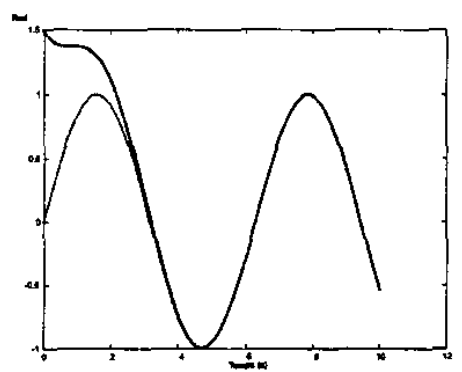

Fig. 3. the response of $q_{1}(t)$ (solid line) and the desired value $\mathrm{q}_{\mathrm{r} 1}(\mathrm{t})=\sin (\mathrm{t})$ (dashed line)

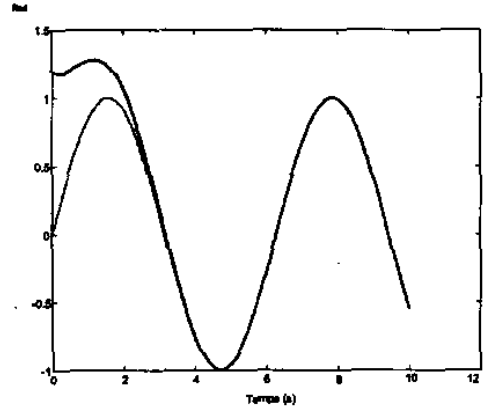

Fig. 4. The response of $q_{2}(t)$ (solid line) and the desired trajectory $q_{r_{2}}(t)=\sin (t)$ (dashed line)

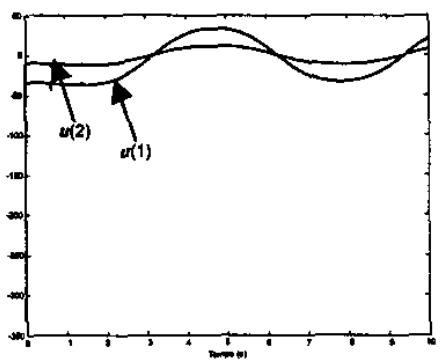

Fig. 5. The applied torques

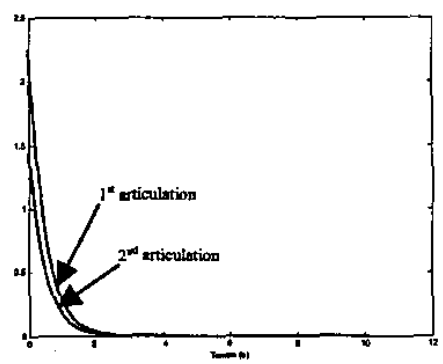

Fig. 6. The quadratic errors

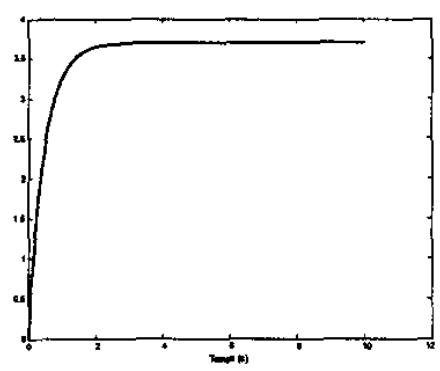

Fig. 7. The quadratic error integral 